\title{
ON SOME FRACTIONAL INTEGRAL INEQUALITIES OF HERMITE-HADAMARD TYPE FOR $r$-PREINVEX FUNCTIONS
}

\author{
ABDULLAH AKKURT AND HÜSEYIN YILDIRIM
}

[Department of Mathematics, Faculty of Science and Arts, University of Kahramanmaraş SütÇÜ İmam, 46000, Kahramanmaraş, TURKeY; hyildir@ksu.edu.tr

* Correspendence: abdullahmat@gmail.com

ABSTRACT: In this paper, we have established Hermite-Hadamard inequalities for $r$-preinvex functions via fractional integrals.

Keywords: integral inequalities; fractional integrals; Hermite-Hadamard inequality; preinvex functions

2010 Mathematics Subject Classification 26D15, 26A51, 26A33, 26A42.

\section{INTRODUCTION}

Let $f: I \subseteq \mathbb{R} \rightarrow \mathbb{R}$ be a convex function defined on the interval $I$ of real numbers and $a, b \in I$ with $a<b$. The following inequality holds

$$
f\left(\frac{a+b}{2}\right) \leq \frac{1}{b-a} \int_{a}^{b} f(x) d x \leq \frac{f(a)+f(b)}{2} .
$$

This double inequality (1.1) is known in the literature as Hermite-Hadamard integral inequality for convex functions. Both inequalities hold in the reversed direction if $\mathrm{f}$ is concave.The inequality (1.1) has been extended and generalized for various classes of convex functions via different approaches, see $[4,7,10,12]$. For several recent result concerning the inequality (1.1) we refer the interested reader to $[1-11,13,15-17,19]$ and references cited therein.

\section{Preliminares}

Let $K$ be an nonempty subset of $\mathbb{R}^{n}$ and let $\eta: K \times K \rightarrow \mathbb{R}^{n}$.

Definition 1. ([24]) Let $u \in K$. We say $K$ is invex at $u$ with respect to $\eta$ if, for each $v \in K$

$$
u+t \eta(v, u) \in K, t \in[0,1] .
$$

$K$ is said to be an invex set with respect to $\eta$ if $K$ is invex at each $u \in K$.

Definition 2. ([14]) The function $f$ on the invex set $K$ is said to be preinvex with respect to $\eta$, if

$$
f(u+t \eta(v, u)) \leq(1-t) f(u)+t f(v), \quad \forall u, v \in K, t \in[0,1] .
$$

Definition 3. ([18]) A positive function $f$ on the invex set $K$ is said to be logarithmically preinvex, if

$$
f(u+t \eta(v, u)) \leq f^{1-t}(u) f^{t}(v)
$$

for all $u, v \in K$ and $t \in[0,1]$. 
Definition 4. ([18]) The function $f$ on the invex set $K$ is said to be preinvex with respect to $\eta$, if

$$
f(u+t \eta(v, u)) \leq M_{r}(f(u), f(v) ; t)
$$

holds for all $u, v \in K$ and $t \in[0,1]$, where

$$
M_{r}(x, y ; t)= \begin{cases}{\left[(1-t) x^{r}+t y^{r}\right]^{\frac{1}{r}}} & , r \neq 0 \\ x^{1-t} y^{t} & , r=0\end{cases}
$$

is the weighted power mean of order $r$ for positive numbers $x, y$.

Definition 5. ([19]) Let $f \in L^{1}[a, b]$. The Riemann-Liouville fractional integral $J_{a^{+}}^{\alpha} f(x)$ and $J_{b^{-}}^{\alpha} f(x)$ of order $\alpha>0$ are defined by

$$
J_{a^{+}}^{\alpha}[f(x)]=\frac{1}{\Gamma(\alpha)} \int_{a}^{x}(x-t)^{\alpha-1} f(t) d t \quad x>a
$$

and

$$
J_{b^{-}}^{\alpha}[f(x)]=\frac{1}{\Gamma(\alpha)} \int_{x}^{b}(t-x)^{\alpha-1} f(t) d t \quad x<b
$$

respectively, where $\Gamma(\alpha)=\int_{0}^{\infty} e^{-u} u^{\alpha-1} d u$ is Gamma function and $J_{a^{+}}^{0} f(x)=J_{b^{-}}^{0} f(x)=$ $f(x)$.

\section{Main Results}

Theorem 1. Let $f: K=[a, a+\eta(b, a)] \rightarrow(0, \infty)$ be an $r$-preinvex functions on the interval of real numbers $K^{\circ}$ (the interior of $K$ ) and $a, b \in K^{\circ}$ with $a<a+\eta(b, a)$. then

$$
\left(J_{(a+\eta(b, a))^{-}}^{\alpha} f\right)(a) \leq \frac{[\eta(b, a)]^{\alpha}}{\Gamma(\alpha)}\left\{f^{r}(b) \frac{r}{\alpha r+1}+f^{r}(a) B\left(\alpha, \frac{1}{r}+1\right)\right\}^{\frac{1}{r}}
$$

holds for $0<r \leq 1$.

Proof. Since $f$ is an $r$-preinvex function and $r>0$, we have

$$
f\left(a+t \eta(b, a) \leq\left[t f^{r}(b)+(1-t) f^{r}(a)\right]^{\frac{1}{r}}\right.
$$

for all $t \in[0,1]$. Then,

$$
\begin{aligned}
\frac{\Gamma(\alpha)}{[\eta(b, a)]^{\alpha}}\left(J_{(a+\eta(b, a))^{-}}^{\alpha}\right)(a) & =\frac{1}{[\eta(b, a)]^{\alpha}} \int_{a}^{a+\eta(b, a)}(u-a)^{\alpha-1} f(u) d u \\
& =\int_{0}^{1} t^{\alpha-1} f(a+t \eta(b, a)) d t \\
& \leq \int_{0}^{1} t^{\alpha-1}\left[t f^{r}(b)+(1-t) f^{r}(a)\right]^{\frac{1}{r}} d t \\
& =\int_{0}^{1}\left[t^{r(\alpha-1)+1} f^{r}(b)+t^{r(\alpha-1)}(1-t) f^{r}(a)\right]^{\frac{1}{r}} d t .
\end{aligned}
$$


Using Minkowski's inequality, we have

$$
\begin{aligned}
& \int_{0}^{1}\left[t^{r(\alpha-1)+1} f^{r}(b)+t^{r(\alpha-1)}(1-t) f^{r}(a)\right]^{\frac{1}{r}} d t \\
& \leq\left\{\left[\int_{0}^{1} t^{\alpha-1+\frac{1}{r}} f(b) d t\right]^{r}+\left[\int_{0}^{1} t^{\alpha-1}(1-t)^{\frac{1}{r}} f(a) d t\right]^{r}\right\}^{\frac{1}{r}} \\
& =\left\{f^{r}(b) \frac{r}{\alpha r+1}+f^{r}(a) B\left(\alpha, \frac{1}{r}+1\right)\right\}^{\frac{1}{r}} .
\end{aligned}
$$

Theorem 1 is proved.

Remark 1. Under conditions of Theorem $1, \alpha=1, r=1$ and $\eta(b, a)=b-a$, we have

$$
\frac{1}{b-a} \int_{a}^{b} f(x) d x \leq \frac{f(a)+f(b)}{2} .
$$

Theorem 2. Let $f, g: K=[a, a+\eta(b, a)] \rightarrow(0, \infty)$ be $r$-preinvex and $s$-preinvex functions respectively on the interval of real numbers $K^{\circ}, a, b \in K^{\circ}$ with $a<$ $a+\eta(b, a)$, then

$$
\begin{aligned}
& \left(J_{(a+\eta(b, a))^{-}}^{\alpha} f g\right)(a) \\
& \leq \frac{1}{2} \frac{[\eta(b, a)]^{\alpha}}{\Gamma(\alpha)}\left\{\left(f^{r}(b) \frac{r}{2 \alpha+r}+f^{r}(a) B\left(\frac{2(\alpha-1)}{r}+1, \frac{2}{r}+1\right)\right)^{\frac{2}{r}}\right. \\
& \left.+\left(g^{s}(b) \frac{s}{2 \alpha+s}+g^{s}(a) B\left(\frac{2(\alpha-1)}{s}+1, \frac{2}{s}+1\right)\right)^{\frac{2}{s}}\right\}
\end{aligned}
$$

holds for $0<r, s \leq 2$.

Proof. Since $f$ is an $r$-preinvex function and $g$ is a $s$-preinvex function, by hypothesis of theorem, we have

$$
f\left(a+t \eta(b, a) \leq\left[t f^{r}(b)+(1-t) f^{r}(a)\right]^{\frac{1}{r}}\right.
$$

and

$$
g\left(a+t \eta(b, a) \leq\left[t g^{s}(b)+(1-t) g^{s}(a)\right]^{\frac{1}{s}}\right.
$$

for $t \in[0,1]$. By using the inequality (3.3) and (3.4), we get

$$
\begin{aligned}
& \frac{1}{[\eta(b, a)]^{\alpha}} \int_{a}^{a+\eta(b, a)}(u-a)^{(\alpha-1)\left(\frac{1}{r}+\frac{1}{s}\right)} f(u) g(u) d u \\
& =\int_{0}^{1} t^{(\alpha-1)\left(\frac{1}{r}+\frac{1}{s}\right)} f(a+t \eta(b, a)) g(a+t \eta(b, a)) d t \\
& \leq \int_{0}^{1} t^{(\alpha-1)\left(\frac{1}{r}+\frac{1}{s}\right)}\left[t f^{r}(b)+(1-t) f^{r}(a)\right]^{\frac{1}{r}}\left[t g^{s}(b)+(1-t) g^{s}(a)\right]^{\frac{1}{s}} d t .
\end{aligned}
$$


Using Cauchy's inequality for (3.5), we have

$$
\begin{aligned}
& \int_{0}^{1}\left[t^{\alpha} f^{r}(b)+t^{\alpha-1}(1-t) f^{r}(a)\right]^{\frac{1}{r}}\left[t^{\alpha} g^{s}(b)+t^{\alpha-1}(1-t) g^{s}(a)\right]^{\frac{1}{s}} d t \\
& \leq \frac{1}{2}\left\{\int_{0}^{1}\left[t^{\alpha} f^{r}(b)+t^{\alpha-1}(1-t) f^{r}(a)\right]^{\frac{2}{r}} d t\right. \\
& \left.+\int_{0}^{1}\left[t^{\alpha} g^{s}(b)+t^{\alpha-1}(1-t) g^{s}(a)\right]^{\frac{2}{s}} d t\right\} \\
& =\frac{1}{2}\left\{I_{1}+I_{2}\right\} .
\end{aligned}
$$

Using Minkowski's inequality for $I_{1}$ and $I_{2}$, we hawe

$$
\begin{aligned}
& I_{1}=\int_{0}^{1}\left[t^{\alpha} f^{r}(b)+t^{\alpha-1}(1-t) f^{r}(a)\right]^{\frac{2}{r}} d t \\
& \leq\left\{\left(\int_{0}^{1} t^{\frac{2}{r} \alpha} f^{2}(b) d t\right)^{\frac{r}{2}}+\left(\int_{0}^{1} t^{\frac{2(\alpha-1)}{r}}(1-t)^{\frac{2}{r}} f^{2}(a) d t\right)^{\frac{r}{2}}\right\}^{\frac{2}{r}} \\
& =\left\{f^{r}(b) \frac{r}{2 \alpha+r}+f^{r}(a) B\left(\frac{2(\alpha-1)}{r}+1, \frac{2}{r}+1\right)\right\}^{\frac{2}{r}}
\end{aligned}
$$

and

$$
\begin{aligned}
& I_{2}=\int_{0}^{1}\left[t^{\alpha+1} g^{s}(b)+t^{\alpha}(1-t) g^{s}(a)\right]^{\frac{2}{s}} d t \\
& \leq\left\{g^{s}(b) \frac{s}{2 \alpha+s}+g^{s}(a) B\left(\frac{2(\alpha-1)}{s}+1, \frac{2}{s}+1\right)\right\}^{\frac{2}{s}} .
\end{aligned}
$$

Combining $I_{1}$ and $I_{2}$ inequalities lead to (3.2). Theorem 2 is proved.

Corollary 1. Under conditions of Theorem $2, r=s=2$, we have

$$
\frac{\Gamma(\alpha)}{[\eta(b, a)]^{\alpha}}\left(J_{(a+\eta(b, a))^{\alpha}}^{\alpha} f g\right)(a) \leq \frac{f^{2}(a)+f^{2}(b)+g^{2}(a)+g^{2}(b)}{2(\alpha+1)} .
$$

Corollary 2. Under conditions of Theorem 2, $\eta(b, a)=b-a$ and $r=s=2$, we have

$$
\frac{\Gamma(\alpha)}{(b-a)^{\alpha}} J_{b^{+}}^{\alpha} f g(a) \leq \frac{f^{2}(a)+f^{2}(b)+g^{2}(a)+g^{2}(b)}{2(\alpha+1)} .
$$

Corollary 3. Under conditions of Theorem 2, $\alpha=1$ and $r=s=2$, we have

$$
\frac{1}{[\eta(b, a)]} \int_{a}^{a+\eta(b, a)} f(u) g(u) d u \leq \frac{f^{2}(a)+f^{2}(b)+g^{2}(a)+g^{2}(b)}{4}
$$

in [18].

Corollary 4. Under conditions of Theorem $2, \alpha=1, \eta(b, a)=b-a$ and $r=s=2$, we have

$$
\frac{1}{[b-a]} \int_{a}^{b} f(u) g(u) d u \leq \frac{f^{2}(a)+f^{2}(b)+g^{2}(a)+g^{2}(b)}{4} .
$$

Corollary 5. Under conditions of Theorem 2 with $\alpha=1, r=s=2$, and $f(x)=$ $g(x)$, we have

$$
\frac{1}{\eta(b, a)} \int_{a}^{a+\eta(b, a)} f^{2}(u) d u \leq \frac{f^{2}(a)+f^{2}(b)}{2}
$$

in [18]. 
Theorem 3. Let $f, g: K=[a, a+\eta(b, a)] \rightarrow(0, \infty)$ be $r$-preinvex and s-preinvex functions respectively on the interval of real numbers $K^{\circ}, a, b \in K^{\circ}$ with $a<$ $a+\eta(b, a)$. If $r>1$ and $\frac{1}{r}+\frac{1}{s}=1$, then

$$
\begin{aligned}
& \left(J_{(a+\eta(b, a))^{-}}^{\alpha} f g\right)(a) \\
& \leq \frac{[\eta(b, a)]^{\alpha}}{\Gamma(\alpha)}\left\{\left(f^{r}(b) \frac{1}{\alpha+1}+f^{r}(a) B(\alpha, 2)\right)^{\frac{1}{r}}\right. \\
& \left.+\left(g^{s}(b) \frac{1}{\alpha+1}+g^{s}(a) B(\alpha, 2)\right)^{\frac{1}{s}}\right\} .
\end{aligned}
$$

Proof. Since $f$ is an $r$-preinvex function and $g$ is a $s$-preinvex function for $t \in[0,1]$, we have

$$
f\left(a+t \eta(b, a) \leq\left[t f^{r}(b)+(1-t) f^{r}(a)\right]^{\frac{1}{r}}\right.
$$

and

$$
g\left(a+t \eta(b, a) \leq\left[g^{s}(b)+(1-t) g^{s}(a)\right]^{\frac{1}{s}} .\right.
$$

From (3.6) and (3.7), we get

$$
\begin{aligned}
& \frac{1}{[\eta(b, a)]^{\alpha}} \int_{a}^{a+\eta(b, a)}(u-a)^{(\alpha-1)\left(\frac{1}{r}+\frac{1}{s}\right)} f(u) g(u) d u \\
& =\int_{0}^{1} t^{(\alpha-1)\left(\frac{1}{r}+\frac{1}{s}\right)} f(a+t \eta(b, a)) g(a+t \eta(b, a)) d t \\
& \leq \int_{0}^{1} t^{(\alpha-1)\left(\frac{1}{r}+\frac{1}{s}\right)}\left[t f^{r}(b)+(1-t) f^{r}(a)\right]^{\frac{1}{r}}\left[t g^{s}(b)+(1-t) g^{s}(a)\right]^{\frac{1}{s}} d t .
\end{aligned}
$$

By virtue of Hölder's inequality, we have

$$
\begin{aligned}
& \int_{0}^{1}\left[t^{\alpha} f^{r}(b)+t^{\alpha-1}(1-t) f^{r}(a)\right]^{\frac{1}{r}}\left[t^{\alpha} g^{s}(b)+t^{\alpha-1}(1-t) g^{s}(a)\right]^{\frac{1}{s}} d t \\
& \leq\left\{\int_{0}^{1}\left[t^{\alpha} f^{r}(b)+t^{\alpha-1}(1-t) f^{r}(a)\right] d t\right\}^{\frac{1}{r}} \\
& +\left\{\int_{0}^{1}\left[t^{\alpha} g^{s}(b)+t^{\alpha-1}(1-t) g^{s}(a)\right] d t\right\}^{\frac{1}{s}} \\
& =\frac{[\eta(b, a)]^{\alpha}}{\Gamma(\alpha)}\left\{\left(f^{r}(b) \frac{1}{\alpha+1}+f^{r}(a) B(\alpha, 2)\right)^{\frac{1}{r}}\right. \\
& \left.+\left(g^{s}(b) \frac{1}{\alpha+1}+g^{s}(a) B(\alpha, 2)\right)^{\frac{1}{s}}\right\} .
\end{aligned}
$$

The proof is done.

Corollary 6. Under conditions of Theorem 2, if $r=s=2$, we have

$$
\begin{aligned}
& \left(J_{(a+\eta(b, a))^{-}}^{\alpha} f g\right)(a) \\
& \leq \frac{[\eta(b, a)]^{\alpha}}{\Gamma(\alpha)}\left\{\sqrt{\left(f^{2}(b) \frac{1}{\alpha+1}+f^{2}(a) B(\alpha, 2)\right)}\right. \\
& \left.\quad+\sqrt{g^{2}(b) \frac{1}{\alpha+1}+g^{2}(a) B(\alpha, 2)}\right\} .
\end{aligned}
$$


Corollary 7. Under conditions of Theorem 2, if $r=s=2, \eta(b, a)=b-a$, we have

$$
\begin{gathered}
\frac{(b-a)^{\alpha}}{\Gamma(\alpha)} J_{b^{+}}^{\alpha} f g(a) \leq\left\{\sqrt{\left(f^{2}(b) \frac{1}{\alpha+1}+f^{2}(a) B(\alpha, 2)\right)}\right. \\
\left.+\sqrt{g^{2}(b) \frac{1}{\alpha+1}+g^{2}(a) B(\alpha, 2)}\right\} .
\end{gathered}
$$

Corollary 8. Under conditions of Theorem 2, if $r=s=2, \eta(b, a)=b-a$ and $\alpha=1$, we have

$$
\frac{1}{b-a} \int_{a}^{b} f(u) g(u) d u \leq \sqrt{\frac{f^{2}(a)+f^{2}(b)}{2}} \sqrt{\frac{g^{2}(a)+g^{2}(b)}{2}} .
$$

Corollary 9. Under conditions of Theorem 2, if $r=s=2$ and $\alpha=1$, we have

$$
\frac{1}{[\eta(b, a)]} \int_{a}^{a+\eta(b, a)} f(u) g(u) d u \leq \sqrt{\frac{f^{2}(a)+f^{2}(b)}{2}} \sqrt{\frac{g^{2}(a)+g^{2}(b)}{2}} .
$$

\section{REFERENCES}

[1] Barani, A.; Ghazanfari, A.G.; Dragomir, S.S.: Hermite-Hadamard inequality for functions whose derivatives absolute values are preinvex. J. Inequal. Appl. 2012, 247 (2012)

[2] Dragomir, S.S.; Pearce, C.E.M.: Selected topics on Hermite-Hadamard inequalities and applications. Victoria University (2000)

[3] Dragomir, S.S.; Pecaric, J.; Persson, L.E.: Some inequalities of Hadamard type. Soochow J. Math. 21, 335-341, (1995)

[4] İşcan, İ.: Hermite-Hadamard's inequalities for preinvex function via fractional integrals and related functional inequalities, Am. J. Math. Anal. 1 (3) (2013) 33-38.

[5] Latif, M.A.; Dragomir, S.S.: Some Hermite-Hadamard type inequalities for functions whose partial derivatives in absloute value are preinvex on the co-oordinates. Facta Universitatis (NIS) Ser. Math. Inform. 28(3), 257-270, (2013)

[6] M.A. Latif, ; Dragomir, S. S.; Momoniat, E.: Some weighted integral inequalities for differentiable preinvex and prequasiinvex functions. RGMIA (2014)

[7] M.A. Noor and K.I. Noor, Generalized preinvex functions and their properties. Journal of Appl. Math. Stochastic Anal., 2006(12736), 1-13, doi:10.1155/JAMSA/2006/12736

[8] M.A. Noor, K.I. Noor, M.A. Ashraf, M.U. Awan and B. Bashir, Hermite-Hadamard inequalities for $h_{\varphi}$-convex functions. Nonl. Anal. Formum. 18, 65-76 (2013)

[9] M.A. Noor, K.I. Noor and M.U. Awan, Hermite-Hadamard inequalities for relative semiconvex functions and applications. Filomat, 28(2), 221-230, (2014)

[10] M.A. Noor, K.I. Noor and M.U. Awan, Generalized convexity and integral inequalities. Appl. Math. Inf. Sci. 9(1), 233-243, (2015)

[11] M.A. Noor, K.I. Noor, M.U. Awan and J. Li, On Hermite-Hadamard type Inequalities for $h$-preinvex functions. Filomat, in press

[12] M.A. Noor and K.I. Noor, Generalized preinvex functions and their properties. Journal of Appl. Math. Stochastic Anal., 2006(12736), 1-13, doi:10.1155/JAMSA/2006/12736

[13] M.A. Noor, Some new classes of nonconvex functions. Nonl. Funct. Anal. Appl. 11(1), 165-171 (2006)

[14] M.A. Noor, K.I. Noor, M.U. Awan and S. Khan, Hermite-Hadamard inequalities for differantiable $h_{\varphi}$-preinvex functions. Arab. J. Math.4:63-76 (2015)

[15] Sarikaya, M.Z.; Set, E.: Özdemir M.E.: On some new inequalities of Hadamard type involving $h$-convex functions. Acta Math. Univ. Comenianae. 2, 265-272 (2010)

[16] Sarikaya, M.Z.; Alp, N.; Bozkurt, H.: On Hermite-Hadamard Type Integral Inequalities for preinvex and log-preinvex functions, Contemporary Analysis and Applied Mathematics, Vol.1, No.2, 237-252, 2013.

[17] Varosanec, S.: On $h$-convexity. J. Math. Anal. Appl. 326, 303-311 (2007) 
[18] Wei-Dong Jiang, Da-Wei Nıu, Feng Qi: Some Fractional Inequalties of Hermite-Hadamard type for $r-\varphi$-Preinvex Functions, Tamkang Journal of Mathematics, Vol. 45, No. 1, 31-38, 2014.

[19] Samko, S.G.; Kilbas, A.A.; Marichev, O.I.: Fractional Integrals and Derivatives, Theory and Applications, Gordon and Breach, Yverdon, Switzerland, 1993

[20] Y.-L. Bi, Hadamard type inequality for $r$-preinvex functions, Math. Pract. Theory, 39 (2009), 190-192. (Chinese)

[21] S. S. Dragomir and S. Fitzpatrick, The Hadamard's inequality for s-convex functions in the second sense, Demonstratio Math., 32 (1999), 687-696.

[22] S. R. Mohan and S. K. Neogy, On invex sets and preinvex functions, J.Math. Anal. Appl., 189 (1995), 901-908; Available online at htp:/dx.doi.org/10.106/jma.195.1057

[23] M. A. Noor, Nonconvex functions and variational inequalities, J. Optim. Theory Appl., 87 (1995), 615-630; Available online at htp:/dx.doi.org/10.107/BF02192137.

[24] T. Weir and B. Mond, Pre-invex functions in multiple objective optimization, J. Math. Anal. Appl., 136(1988), 29-38; Available online at htp:/dx.doi.org/10.1016/02-247X(8)9013-8.

[25] M. A.Noor,On Hermite-Hadamard integral inequalities for product of two nonconvex functions, J. Adv.Math. Studies, 2 (2009), 53-62.

[26] W.-D. Jiang, Hermite-Hadamard type inequality for $r-\varphi$-convex functions, Búdĕngshì Yānjiu Tõngxún (Communications in Studies on Inequalities), 17 (2010), 387-394. (Chinese)

(C) 2016 by the authors; licensee Preprints, Basel, Switzerland. This article is an open access article distributed under the terms and conditions of the Creative Commons by Attribution (CC-BY) license (http://creativecommons.org/licenses/by/4.0/). 